\title{
Covid-19: Surviving the long road ahead
}

\author{
Fiona Godlee editor in chief
}

The BMJ

More than four months after the first cases in China, we know a lot about covid-19. But much is still uncertain, especially about the clinical course and long term effects of this multisystem disease. Mike Roberts and colleagues describe the hyperinflammatory response that can affect the lungs, heart, kidneys, nerves, muscles, gut, and brain (https://bit.ly/ 2xBn7M6). They encourage clinicians to actively seek signs of organ involvement beyond the respiratory tract and to consider early renal support to avoid acute kidney injury.

Dan Martin and his intensive care colleagues (https://bit.ly/ 3c2JOrA) have had largely to discard their protocols developed for acute respiratory distress syndrome (ARDS). The relative ease of ventilation and high incidence of acute kidney injury have meant lower ventilatory pressures and more intravenous fluid. But Susan Wilcox writes that the lung pathology is not dissimilar to ARDS (doi:10.1136/bmj.m1786). Arguments for and against early intubation remain unresolved because of a lack of good evidence, she says, and risks to staff from aerosol transmission must be weighed alongside the needs of each patient.

While most people have mild, if any, symptoms and uncomplicated recoveries, some have prolonged illness. Paul Garner's has continued for weeks, with a shifting array of troubling symptoms: extreme fatigue, headaches, muscle pains, breathlessness, "like an advent calendar, every day there was a surprise, something new" (https://bit.ly/3b6z8XO). He wants greater understanding and support for himself and others.

Understanding and support are urgently needed for people discharged from intensive care, writes Roger Boyes, who feels a sense of abandonment, struggling at home after five weeks in hospital (doi:10.1136/bmj.m1813). We can expect a "tsunami of rehabilitation needs," reports Jacqui Thornton (doi:10.1136/ bmj.m1787), as the many patients discharged after longer than usual stays in intensive care units have to deal with muscle wastage, sleep disorders, fatigue, memory problems, anxiety, depression, and post-traumatic stress disorder. GPs will need help in responding to this new wave of problems so they can support patients and their families through the long haul. Hospitals are developing "intensive aftercare" services, but these will have to compete for funds. Thornton quotes one physiotherapist: "Why invest so much ICU time and resource to save a life, to then leave a patient with debilitating symptoms and a family floundering?"

The overwhelming focus on covid-19 has led to neglect of many other conditions, so plans to restart regular services are welcome (doi:10.1136/bmj.m1793). We must not, however, lose the many service innovations inspired by the covid response. Describing the "spectacular acceleration" of transformation in ophthalmology, Alastair Denniston and coauthors want no going back to the old ways (https://bit.ly/3fmWr2G).

But Clare Gerada and Caroline Walker find that they and many others are struggling to absorb the enormous changes of the past few months (https://bit.ly/3bbaK77). They warn that this is not a marathon but multiple marathons run one after the other and that for many clinicians the initial eagerness to "get stuck in" has been replaced by intense and overwhelming "covid fatigue." Good pacing, refreshment, and support will be key to surviving the long road ahead. 